Revista de

Estudios

Kantianos

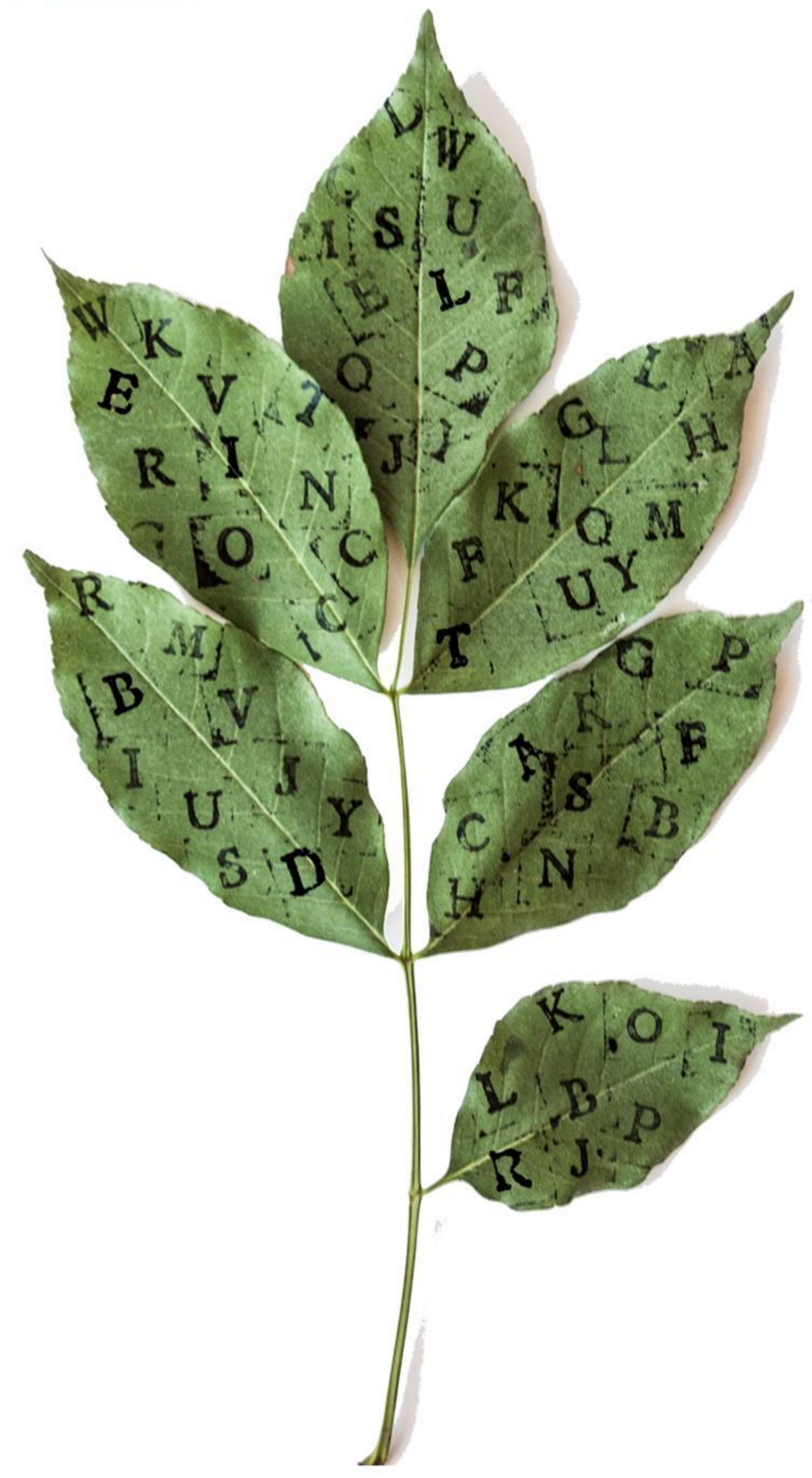


Revista de Estudios Kantianos 


\section{Revista de Estudios Kantianos}

Publicación internacional de la Sociedad de Estudios Kantianos en Lengua Española Internationale Zeitschrift der Gesellschaft für Kant-Studien in Spanischer Sprache International Journal of the Society of Kantian Studies in the Spanish Language

\section{Dirección}

Pedro Jesús Teruel, Universitat de València

pedro.teruel@uv.es

Hernán Pringe, CONICET-Universidad de Buenos Aires/

Universidad Diego Portales, Santiago de Chile

hpringe@gmail.com

\section{Secretario de edición}

Fernando Moledo, CONICET-Universidad de Buenos Aires

fernandomoledo@filo.uba.ar

\section{Secretaria de calidad}

Marcela García, Universidad de Morelia, México

garciar.marcela@gmail.com

\section{Editores científicos}

Jacinto Rivera de Rosales, UNED, Madrid

Claudia Jáuregui, Universidad de Buenos Aires

Vicente Durán, Pontificia Universidad Javeriana, Bogotá

Julio del Valle, Pontificia Universidad Católica del Perú, Lima

Jesús Conill, Universitat de València

Gustavo Leyva, Universidad Autónoma de México, México D. F.

María Xesús Vázquez Lobeiras, Universidade de Santiago de Compostela

Wilson Herrera, Universidad del Rosario, Bogotá

Pablo Oyarzun, Universidad de Chile, Santiago de Chile

Paula Órdenes Azúa, Universität Heidelberg 


\section{Comité científico}

Juan Arana, Universidad de Sevilla

Reinhardt Brandt, Philipps-Universität Marburg

Mario Caimi, Universidad de Buenos Aires

Monique Castillo, Université de Paris-Est

Adela Cortina, Universitat de València

Bernd Dörflinger, Universität Trier

Norbert Fischer, Universität Eichstätt-Ingolstadt

Miguel Giusti, Pontificia Universidad Católica del Perú

Dulce María Granja, Universidad Nacional Autónoma de México

Christian Hamm, Universidad Federal de Santa María, Brasil

Dietmar Heidemann, Université du Luxembourg

Otfried Höffe, Universität Tübingen

Claudio La Rocca, Università degli Studi di Genova

Juan Manuel Navarro Cordón, Universidad Complutense, Madrid

Carlos Pereda, Universidad Nacional Autónoma de México

Gustavo Pereira, Universidad de la República, Uruguay

Ubirajara Rancan de Azevedo, Universidade Estadual Paulista, Brasil

Margit Ruffing, Johannes Gutenberg-Universität Mainz

Gustavo Sarmiento, Universidad Simón Bolívar, Venezuela

Sergio Sevilla, Universitat de València

Roberto Torretti, Universidad Diego Portales, Santiago de Chile

Violetta Waibel, Universität Wien

Howard Williams, University of Aberystwyth

Allen W. Wood, Indiana University

Diseño, editor de estilo y maqueta

Josefa Ros Velasco, Universidad Complutense de Madrid

\section{Entidades colaboradoras}

Sociedad de Estudios Kantianos en Lengua Española (SEKLE)

Departament de Filosofia de la Universitat de València

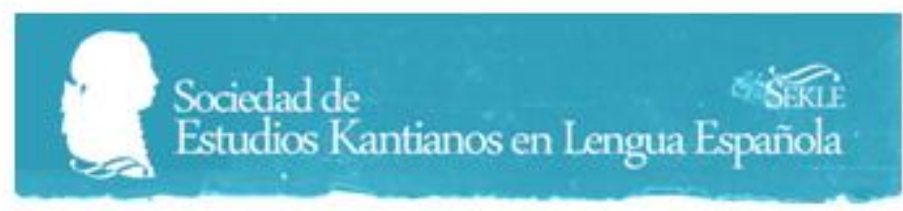




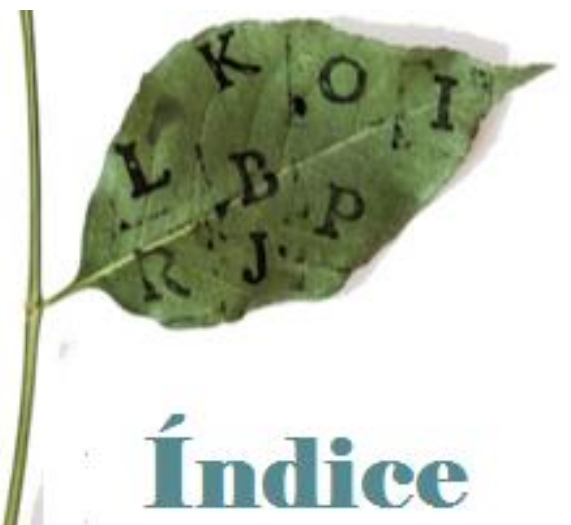

\section{Artículos}

9 Pensar como operación - Acerca de los presupuestos e implicaciones de la lógica formal moderna

Max Gottschlich

DOI 10.7203/REK.2.1.10059

20 Los conceptos kantianos de "facultad" y de "mente" frente a la lectura epigenética Antonino Falduto

DOI 10.7203/REK.2.1.10011

29 Arqueología filosófica y hermenéutica. Apuntes sobre la interpretación de la historia de la filosofía en Kant

José García Gómez del Valle

DOI 10.7203/REK.2.1.8811

48 Continuidad y novedad en la concepción histórica de Kant en El conflicto de las facultades

Natalia Lerussi

DOI 10.7203/REK.2.1.9892

60 It's impossible to will to be punished? Exploring consensual way out of the Kantian dilema Matías Parmigiani

DOI 10.7203/REK.2.1.8813

\section{Recensiones}

89 Immanuel Kant (trad. Alba Jiménez): Lecciones de filosofía moral Mrongovius II.

Salamanca, Sígueme, 2017, 160 pp. ISBN: 978-8-4301-1954-7

Pablo Veraza Tonda

DOI 10.7203/REK.2.1.9990 
91 Faviola Rivera Castro: Virtud, Felicidad y Religión en la Filosofía Moral de Kant. México, Instituto de Investigaciones Filosóficas-UNAM, 2014, 328 pp. ISBN: 978-607-02-4788-0

Gustavo Leyva

DOI 10.7203/REK.2.1.9992

93 Vicente de Haro Romo: Duty, Virtue and Practical Reason in Kant's Metaphysics of Morals. Hildesheim/Zürich/New York, Georg Olms Verlag, 2015, 342 pp. ISBN: 978-3487-15348-3

Eduardo E. Charpenel

DOI 10.7203/REK.2.1.9998

96 Paolo Grillenzoni: Kant e la scienza (1755-1760). Roma, Aracne, 2016, 575 pp. ISBN: 9788-854-89523-2

Daniele Savino

DOI 10.7203/REK.2.1.10054

100 Nuria Sánchez Madrid: A Civilização como destino. Kant e as formas da reflexão.

Florianópolis, Nefiponline, 2016, 288 pp. ISBN: 978-85-99608-16-6

Josefa Ros Velasco

DOI 10.7203/REK.2.1.9801

103 Laura Anna Macor: Die Bestimmung des Menschen (1748-1800): Eine Begriffsgeschichte. Forschungen und Materialien zur deutschen Aufklärung. Abteilung II: MonographienFMDA II, 25. Stuttgart-Bad Cannstatt, Frommann-Holzboog, 2013, 432 pp. ISBN: 978-37728-2615-3

Daniel Schwab

DOI 10.7203/REK.2.1.9989

\section{Informes}

109 Rezension zur Internationalen Tagung "Teleologische Reflexion in Kants Philosophie" Anna Pickhan (geb. Brechtel)

DOI 10.7203/REK.2.1.9993

\section{Entrevistas}

112 Entrevista a Pablo Muchnik

Fernando Moledo

DOI 10.7203/REK.2.1.10064

\section{Convocatorias y normas para autores}

118 Kant en México

DOI 10.7203/REK.2.1.10085

119 II Premio Kant para Jóvenes Investigadores e Investigadoras DOI 10.7203/REK.2.1.10086 
120 IV Congreso Internacional de la SEKLE, Valencia, 2018 DOI 10.7203/REK.2.1.10088

121 Normas para autores DOI 10.7203/REK.2.1.10091 


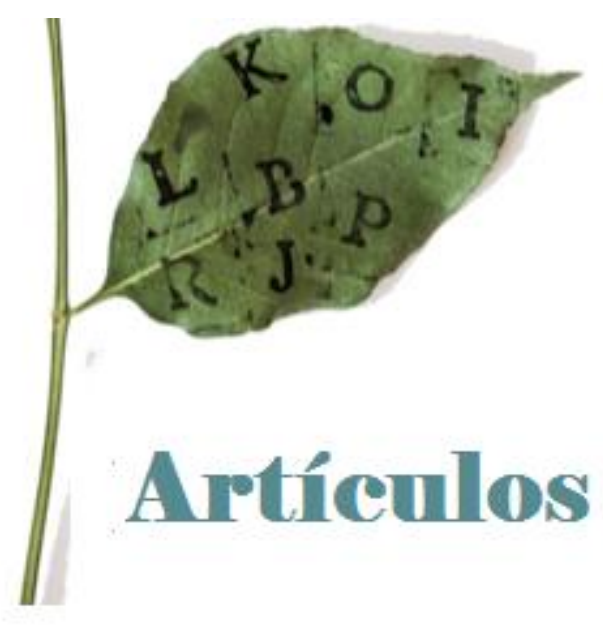




\title{
Continuidad y novedad en la concepción histórica de Kant en El conflicto de las facultades
}

NATALIA LERUSSI ${ }^{1}$

\begin{abstract}
Resumen
En el trabajo analizamos algunas continuidades y novedades fundamentales en la concepción kantiana de la historia en el texto El conflicto de las facultades (1798) en comparación con su perspectiva histórica previa, fundamentalmente, de Idea para una historia universal en clave cosmopolita. Mostramos que, en líneas generales, Kant conserva el marco teórico a partir del cual explica el desarrollo histórico en sus textos anteriores, es decir, los conceptos de disposiciones humanas y de circunstancias externas de desenvolvimiento de dichas disposiciones. Sin embargo, en 1798 modifica el tipo de disposiciones que cumplen el rol explicativo en el desarrollo histórico pues ahora es la disposición moral y no, como antes, las disposiciones no morales aquello que motorizan el progreso histórico.
\end{abstract}

Palabras clave: Historia, Progreso, Disposiciones, Kant

\section{Continuity and Novelty in Kant's Historical Conception in The Conflict of the Faculties}

\begin{abstract}
In the paper I analize some fundamental lines of continuity and novelties in Kant's conception of history in the text The Conflict of the Faculties (1798) in comparison with hisprevioushistoricalperspective, specially in Idea for a Universal Historywith Cosmopolitan Purpose. I show that Kant keeps the theoretical framework through which he explains historical development in his earlier works, that is to say, the concepts of human dispositions and external circumstances. However in 1798 he modifies the kind of dispositions that have the explanatory role in history since now the moral disposition and not like before, the no moral onesis that which motorizes human progress.
\end{abstract}

Key Words: History, Progress, Dispositions, Kant

Introducción

En la segunda parte de El conflicto de las facultades (El conflicto) "Reiteración de la pregunta: Si el género humano se halla en continuo progreso hacia lo mejor" ("Reiteración de la pregunta"), Kant plantea nuevamente la cuestión de la posibilidad del discurso histórico, bajo la pregunta "¿Cómo es posible una historia a priori?". ${ }^{2}$

\footnotetext{
${ }^{1}$ CONICET-UBA

${ }^{2}$ Reinhard Brandt (2003: 121) se pregunta en relación a qué otra pregunta de cuál otro texto debemos remitir el texto "Reiteración de la pregunta". Según su conjetura, la misma se refiere "claramente" a la pregunta que Kant se hace en la sección tercera de Teoría y práctica: En torno al tópico: esto vale para la teoría, pero no sirve para la práctica (Teoría y práctica): “¿Hay que amar al género humano en su totalidad o es éste un objeto que se ha de contemplar con enojo?" (TP AA, VIII, 307). La respuesta a esta pregunta, según señalaría Kant, depende de la respuesta a otra: “¿Hay en la naturaleza humana disposiciones de las que se pueda desprender que la especie progresará siempre a mejor...?" Ibidem. De la opinión de que "Reiteración de la pregunta" debe remitirse al texto de 1793 también es Kleingeld (1995: 68); (2009: 171-186).
} 
Algunos investigadores han afirmado que en El conflicto Kant desarrolla una concepción nueva de la historia y/o del progreso histórico. Así, hace algunas décadas Jean-François Lyotard (1994 [1986]: 59) y Hannah Arendt (2003 [1982]: 37) subrayaban, cada uno a su manera, una diferencia sustantiva entre la concepción histórica de Kant de la década de los 80's expuesta ejemplarmente en Idea de una historia universal en sentido cosmopolita (Idea) y aquélla de los 90's, especialmente, de El conflicto. Según ellos, la primera concepción histórica de Kant estaría articulada a través de una teleología fuerte y los conceptos de "intención de la naturaleza", "providencia" e "hilo conductor". En los desarrollos posteriores, sin embargo, estos conceptos habrían sido desplazados por una matriz más cercana a la estética, esto es, por la perspectiva del "espectador" y los conceptos de "signo histórico", "entusiasmo", "enjuiciamiento", etc. ${ }^{3}$ En una líneainterpretativa cercana, aunque centrada en la figura del "actor" (político) que se revela en la segunda parte de El conflicto,se ubican algunos intérpretes más recientes. Así, por ejemplo, Brandt (2003: 119) afirma que "hasta 1795 [inclusive], aquello que conduce a la humanidad hacia el progreso, es llamado Naturaleza o Providencia, cuyas intenciones sólo el filósofo escolar puede descifrar, ahora [es decir, en $E l$ conflicto] es la acción colectiva misma del hombre aquello que es descubierto como lo que nos orienta hacia el progreso". Asimismo, Perez (2014: 158) afirma que "en 1784 la historia de la humanidad es la historia de un propósito de la naturaleza. En 1798 la historia del género humano es la historia de un sujeto colectivo que actúa con entusiasmo en la realización de las ideas de la razón práctica". ${ }^{4}$ Tanto para Brandt como para Perez en la concepción histórica de Kant de finales de los 90's el pueblo (o la acción colectiva del hombre) se define como el actor del progreso histórico en desmedro de la agencia que la naturaleza o providencia parecía tener en las reflexiones kantianas sobre la historia del período 1784-1795. Constatamos, así, un acuerdo creciente entre los especialistassobre el cambio de posición de Kant en los (tardíos) 90's y sobre la novedad de la reflexión histórica de El conflicto. ${ }^{5}$ En el presente trabajo nos interesa participar en esta discusión a partir de un análisis de lascontinuidades y novedades quenos ofrece el marco teórico general mediante el cual Kant piensa la historia. Así, aportaremos nuevos elementos que iluminan el cambio de

\footnotetext{
${ }^{3}$ Una visión diferente, contemporánea a la de estos autores, es defendida, en 1983, por Michel Foucault (1996: 71-82) quien encuentra una superposición, más que una evolución o cambio, entre diferentes concepciones de la historia de Kant entre 1784 y 1798. Así, vincula fuertemente el texto "¿Qué es la ilustración?" de 1784 -el mismo año en el que Kant publica Idea y en el que se encontraría una visión de la historia "teleológica"- con la segunda parte del texto El conflicto. En ambos textos Kant plantearía el problema del "signo histórico" o signo de la época, las preguntas fundamentales por la actualidad y la revolución que, según el autor (Foucault 1996: 82), definen la tarea de la filosofía (en tanto moderna) bajo la nómina de "ontología del presente" u "ontología de nosotros mismos" y en la cual incluye a su propia filosofía

${ }^{4}$ Véase, además: Perez (2006: 76-77; 101-102).

5 Aunque Ana Marta Gonzalez sostiene que "a través de los años la perspectiva de Kant sobre la historia no cambió de forma significativa" (Gonzalez, 2014: 266), matiza su posición, en concordancia con esta línea de interpretación, cuando señala que en $E$ conflicto - en contraste con Idea - Kant "parece ser más consciente de las dificultades involucradas en la adopción de la perspectiva de la providencia..." "mientras que en Idea para una historia universal en clave cosmopolita Kant basa su concepción general de la historia, exclusivamente, en un concepto teleológico de naturaleza [...] en Reiteración de la pregunta explícitamente trata con la historia de la libertad" (Gonzalez, 2014: 281). Asimismo, tanto para Flikschuh (2007: 239) como para Rodriguez Aramayo (2016: 404) la concepción histórica tardía de Kant (de 1798) se desenmarca de la concepción teleológica de la naturaleza o basada en la providencia presente en su concepción histórica previa, llegan, incluso, a afirmar, en los términos de la primera intérprete, que en El conflicto "no hay referencia ni a la providencia ni a propósito alguno de la naturaleza" (parece suavizar esta posición en Filkschuh, 2009: 108-109). Ambos comportan la visión, en las palabras del segundo según la cual "todo indica que el vocabulario de la providencia, abundante en los opúsculos de Kant sobre filosofía política o filosofía de la historia, es reemplazada, progresivamente, por un recurso a las leyes de la naturaleza como un tipo de esquema sensible o, incluso un tipo [el autor nos remite aquí a CPrR 5:43] de ley del derecho". Por otro lado, Kleingeld (1995: 82-83; cf. además: 31, 49, 50, 64) también reconoce un cambio de posición en los 90's, aunque a partir del texto Teoría y práctica de 1793. Según esta intérprete tanto en Idea para una historia universal como en la Crítica de la facultad de juzgar (1790) Kant habría defendido una "filosofía de la historia" "desde un punto de vista teórico" pero desde 1793 y hasta 1795, esto es, en Teoría y prácticay en Hacia la paz perpetua habría abogado por una concepción dela historia "desde el punto de vista práctico". Sin embargo, defiende que, en El conflicto,la concepción histórica de Kant sufre una especie de retroceso inexplicable, pues allí la historia sería comprendida "no sólo desde una intención práctica sino también [...] en consideración a lo teórico". De esta manera, Kleingeld (1995: 180), se ve obligada a deci que en el texto de 1798 se encuentran "considerables inconsistencias y dificultades" y, por lo tanto, que "sería metodológica mente insostenible suponer simplemente que Kant aquí no ha sido dogmático". Esta consecuencia de la lectura de Kleingeld nos parece muy insatisfactoria.
} 
posición en la concepción histórica de Kant en el texto de 1798, aunque, veremos, el mismo se produce dentro de una misma familia conceptual. ${ }^{6}$

¿Cuáles son los conceptos centrales que Kant utiliza en 1798 para explicar el decurso histórico? ¿Es nuevo el uso que hace Kant de esos conceptos? ¿Cuáles son los conceptos fundamentales mediante los cuales Kant desarrolla su concepción de la historia antes de 1798? El hecho de que Kant no use aquí, recurrentemente, el concepto de una "providencia", que supone fines e intenciones en la naturaleza por fuera de los seres y procesos que actúan y se desarrollan -a la que llamaremos "concepción trascendente" del progreso histórico- no implica que Kant haya abandonado el marco teórico general mediante el cual comprende la historia en los textos previos a 1798. Como hemos mostrado en otro trabajo (Lerussi, 2015) y veremos sumariamente abajo Kant utiliza en su comprensión de la historia humana, paradigmáticamente, en Idea para una historia universal en clave cosmopolita (Idea) un modelo de desarrollo histórico que inscribe los fines o tendencias (de mejoramiento) en una fuerza interna y formadora de la naturaleza humana, visión que podemos llamar, por oposición, "concepción inmanente" del progreso histórico. ${ }^{7}$ Esto significa, específicamente,que el desarrollo histórico o cultural se explica a partir de los conceptos de "disposiciones" inscriptas en la naturaleza humana y de las "circunstancias externas" que favorecen u obstaculizan el despliegue de dichas disposiciones. ${ }^{8}$

Como veremos en este trabajo, en El Conflicto Kant continúa defendiendo, en líneas generales, un marco teórico semejante al previo, una concepción inmanente de la historia según la cual son, principalmente, los conceptos de "disposiciones" inscriptas en la naturaleza humana y las "circunstancias" externas (u "oportunidad") aquellos quedan cuenta del desarrollo histórico. Ahora, aunque Kant mantenga una misma concepción, realiza modificaciones importantes en la argumentación. Antes de 1798, paradigmáticamente en Idea, aquello que permite comprender el desarrollo histórico son las disposiciones no morales de los hombres: son estas disposiciones las que explicarían el despertar final de la disposición moral. Por el contrario, en 1798 las disposiciones no morales no juegan papel alguno en la argumentación. El progreso del género humano hacia lo mejor se asegura a través de la prueba de que hay una disposición moral en el género humano. Una consecuencia de este giro en la argumentación que comentaremos, muy brevemente y como corolario, al final del trabajo atañe a la estructura de la historia o del progreso. Hasta 1798, paradigmáticamente en Idea, el progreso es el resultado de una acumulación continua de experiencias culturales y civilizatorias pasadas, en El conflicto, el "progreso" es la interrupción del curso del tiempo a partir de la irrupción (por revolución o reforma) de la forma política que esjusta y buena por sí misma, la república.

\footnotetext{
${ }^{6}$ En el presente trabajo presentamos una línea argumental principal que da cuenta de algunas de las continuidades y novedades del texto de 1798 sin pretender ser exhaustivos. Hay un cambio importante en la argumentación, mencionado por Brandt (2003 121),que vale la pena indicar: la condición de la pre-existencia de un orden internacional justo (en la forma de un Estado mundial (1784) o de federación de Estados (1795)) para la institución de órdenes civiles internos justos o repúblicas no tiene rol alguno en el texto de 1798 lo que hace pensar que Kant modificó el sentido de la dependencia entre los dos órdenes: ahora (en 1798) dependería de la institución de repúblicas (y, por lo tanto, del pueblo) la conformación de un orden de derecho internacional justo y, no al revés.

${ }_{7}^{7}$ En el artículo, que abajoretomaremos, hemos mostrado no sólo que en Idea Kant defiende una concepción de la historia que denominamos "inmanente", sino además que esta concepción de la historia es utilizada por Kant también en el marco de su historia natural, en su concepción de la filogénesis de las especies y que coincide con su concepción de la "epigénesis". Véase nuestro artículo: Lerussi, 2015

${ }^{8}$ Otros intérpretes también defienden que uno de los conceptos claves para comprender la concepción histórica de Kant, en líneas generales, es el de “disposición” (natural u originaria). Véase: Lebrun (2009); Wood (1999: 208; 2009: 113); Kleingeld (1995: 126, 194, 214).
} 


\section{Disposiciones del género humano y circunstancias externas en El conflicto de las Facultades}

Kant señala en El conflicto que el progreso hacia lo mejor no debe ser esperado del "curso de las cosas de abajo hacia arriba" (SF AA, VII, 092), esto es, del mejoramiento de los hombres tomados individualmente a través de la educación, ${ }^{9}$ sino "de arriba hacia abajo". Esto es:

Pero, como siempre son hombres los que deben realizar esta educación, hombres que han debido ser educados para esa finalidad, es necesario, teniendo en cuanta la flaqueza de la naturaleza humana y la contingencia de los acontecimientos que favorecen tal resultado, poner la esperanza de su progreso sólo en una sabiduría que viene de arriba (in einer Weisheit von oben herab) (que se llama Providencia cuando es invisible para nosotros), como condición positiva. SF AA, VII, 093.

Esta cita indica que, en El conflicto, Kant no abandona, como afirman algunos intérpretes, lo que en la Introducción denominamos "concepción trascendente" de la historia. Una concepción semejante de la historia se encuentra aquí sugerida, aunque debamos reconocer que no tiene el peso que tenía en los textos previos ni tampoco que sea posible extraer del contexto qué quiere decir Kant cuando señala que la esperanza en el progreso hacia lo mejor debe ponerse en una "sabiduría que viene de arriba", como de una "condición positiva". ${ }^{10}$ Dejamos deliberadamente en suspenso una explicación de la concepción trascedente de la historia presente en El conflicto, puesno es un objetivo del presente trabajo probar que Kant defiende -y en qué medida lo hace- una concepción semejante de la historia aquí o en otro contexto. Lo que buscamos mostrar es que Kant defendió en 1798, como también lo había hecho, según veremos, en 1784, una concepción inmanente de la historia.

Ahora bien, como el filósofo indica en el párrafo que copiamos arriba, dado que el educador de los hombres ha debido ser educado por otro hombre bajo circunstancias contingentes no puede, por sí solo, garantizar aquella educación que conduce a la humanidad hacia el desarrollo de su disposición moral dormida. De allí que señale que la esperanza en el progreso hacia lo mejor debe ponerse en una "sabiduría que viene de arriba". Esta expresión se aclara si la tomamos en su acepción "negativa", según continua el argumento, por la cual aquello que "puede ser esperado (erwartet werden kann) de arriba" es también lo que "puede esperarse y exigirse (erwartet und gefordert werden kann) [de los hombres] para el avance de este fin" (SF AA, VII, 093). Si la cuestión del progreso se decide a partir de aquello que los hombres no sólo deben, sino también pueden hacer como especie tomada colectivamente (y no, distributivamente, como es el caso de la educación), cabe suponer que aquélla

\footnotetext{
${ }^{9}$ Kant no rechaza de plano que el progreso dependa de una buena educación. No obstante, enfatiza que el plan que parte del curso de las cosas "de abajo hacia arriba" hace "más difícil" esperar (hoffen) el progreso. Así: "Esperar (erwarten) que por medio de la formación (Bildung) de la juventud, bajo la dirección familiar y luego de las escuelas [...] con una cultura intelectual y moral reforzada por la doctrina religiosa, se llegará finalmente no sólo a educar buenos ciudadanos, sino a educar para el bien [...] es un plan del que difícilmente se puede esperar (schwerlich hoffen läßt) el éxito deseado" (SF AA, VII, 092). Kant menciona dos razones por las cuales la educación de los hombres sería insuficiente, dada las circunstancias, para despertar la disposición moral de los hombres. Esto es, según la primera dificultad, el pueblo considera que los costos de la educación de los jóvenes no deben recaer sobre él sino sobre el Estado, pero los Estados no republicanos no tienen dinero para la educación pues requieren todo para la guerra. De esta primera dificultad se sigue una segunda pues si la educación no es diseñada según un plan meditado desde e poder superior del Estado que responda a sus propósitos, la misma carece de coherencia alguna (véase: SF AA, VII, 093). Los ciudadanos que costean su propia educación, son formados de acuerdo a planes y criterios educativos y políticos que pueden ir en desmedro del progreso. En consecuencia, en la medida en que la cuestión de la educación se enfrenta a serios obstáculos, no podemos confiarnos que será precisamente ella el medio de mejoramiento de la especie.

${ }^{10}$ Pauline Kleingeld (1995: 84) señala que el filósofo se refiere aquí "a un orden de una sabiduría suprema (divina) [...] que gobierna el mundo" "que implica una modificación fundamental del punto de vista histórico" de Kant, al menos, como "sabiduría positiva". En este contexto, la intérprete señala que Kant también puede estar refiriéndose al Estado (véase: ibídem). P. Kleingeld, sin embargo, no fundamenta ninguna de las dos tesis. Con la expresión "de arriba hacia abajo" como sabiduría en sentido "positivo" es probable que Kant no esté mentando, como supone P. Kleingeld, a un ser divino que gobierna el mundo, sino a la razón en sentido práctico como fuerza capaz de actuar según un principio propio y de producir positivamente las condiciones para el desarrollo moral pleno de la humanidad. Agradezco al Prof. Volker Gerhardt haberme sugerido esta interpretación de la enigmática expresión "sabiduría que viene de arriba" como "condición positiva" anotada aquí por Kant. Con todo, el texto no permite más que conjeturas. Añadimos que, si Gerhardt tuviera razón, entonces, lo que hemos llamado "concepción trascendente" de la historia no implica necesariamente premisas teológicas, pues podría estar sostenida sobre una concepción "sustantiva" de la razón práctica.
} 
atañe a lo que puede la naturaleza humana, esto es, según veremos, a la naturaleza "disposicional" de nuestra especie.

El problema fundamental de "Reiteración de la pregunta", aquello que se intenta saber, es si el devenir futuro ${ }^{11}$ de la especie humana como género moral constituye un progreso hacia lo mejor o no. Puesto que la experiencia no nos permitiría inferir cómo se comportará nuestra especie en el futuro (dado que los hombres son seres libres) y que la vía adivinatoria ("una comunicación y ampliación sobrenaturales de la perspectiva del futuro". SF AA, VII, 079) no es posible, parece clausurado un saber sobre el futuro tanto por la vía natural como por la sobrenatural. Kant, sin embargo, abre una tercera alternativa. Hacia el final del apartado cuarto, Kant concluye que podríamos prever el futuro del acontecer humano -en el sentido de un progreso hacia lo mejor- si pudiéramos otorgarle al hombre una "invariable buena voluntad, aunque limitada". Así:

Si se pudiera atribuir al hombre una voluntad innata e invariablemente buena, aunque limitada, podría predecir con certeza este progreso de su especie hacia lo mejor; pues este progreso se referiría a una circunstancia que él mismo puede producir. SF AA, VII, 084.

Nótese que aquello que se debe probar no es que hay en el hombre una voluntad sujeta a la ley moral -problema que Kant ha discutido y resuelto en la Crítica de la razón práctica- o incluso que habría un aumento "creciente de la moralidad de la intención" (SF AA, VII, 091). La prueba que se busca no puede coincidir con aquella que determina el carácter moral del género humano y esto no sólo porque dicho carácter se prueba suficientemente a partir de la conciencia de la ley moral, sino además porque el mismo no permite predecir el modo como los hombres actuarán, de hecho, en el futuro. ${ }^{12}$ La conciencia de la ley en el hombre determina cómo debe actuar él en cualquier circunstancia pero nunca cómo lo hará efectivamente. ${ }^{13}$ Por eso, aquello que el argumento quiere probar es si los hombres tienen una tendenciadispuesta a trabajar a favor de crear las circunstancias del progreso y, por lo tanto, que se inscribe en la naturaleza humana una disposición hacia la moralidad. ${ }^{14}$ En El conflicto, Kant es muy explícito sobre el alcance de dicha disposición pues se trata de una tendencia al mejoramiento de las condiciones morales jurídico-políticas (ámbito del derecho) y no a una tendencia hacia el aumento de la moralidad en el sentido de la buena intención de los hombres (ámbito de la ética). ${ }^{15}$ Esto significa que si hay progreso "[habría] un aumento de los efectos de la legalidad de sus actos [del hombre] conforme al deber cualquiera que sea la razón que los determine" (SF AA, VII, 091, las itálicas son nuestras).

Así, en la sección quinta, Kant defiende que, aunque no puedan darse pruebas empíricas del progreso, como ya hemos señalado, debe haber algún hecho en la experiencia que nos permita inferir indirectamente, como "signo histórico", al menos, la "posibilidad del progreso" mediante la apelación a un cierto "talante" o "facultad" del género humano (en beneficio del derecho). De este modo:

\footnotetext{
${ }_{11}^{11}$ Aunque también el pasado es objeto de discusión en El conflicto en los pasajes: SF AA, VII, 084; 088.

${ }^{12}$ Esto lo señalaría expresamente Kant en la sección cuarta del texto titulada "Con la experiencia no se puede resolver directamente el problema del progreso", véase: SF AA, VII, 083.

${ }^{13}$ En Teoría y prácticaencontramos, no obstante, un único pasaje en los textos dedicados a la historia universal en el cual Kant pretende demostrar el progreso futuro de la humanidad a partir de "un deber innato" del hombre a contribuir en el mismo. Véase: TP AA, VIII, 309. Pero que dicho argumento no es suficiente se evidencia en el mismo texto cuando Kant señala que, en relación al progreso moral de la especie confía, además de en el deber, en la naturaleza de las cosas "que lleva por la fuerza a donde no se quiere de buen grado (fata volentem ducunt, nolentem trahunt)". TP AA, VIII, 313. También: TP AA, VIII, 310. Esta última tesis se halla nuevamente en Hacia la paz perpetua: ZeF AA, VIII, 365. Katrin Flikschuh (2007) se ha dedicado a estudiar específicamente la tensión entre aquellas dos afirmaciones y defiende que Kant no logra resolverla en el marco de este texto (según la intérprete, recién en la Doctrina del derecho, primera parte de la Metafísica de las costumbres (1797), a través del principio de derecho y en desmedro de una concepción teleológica de la naturaleza, Kant ofrece una perspectiva consistente del progreso histórico).

${ }^{14}$ La disposición moral o "disposición para la personalidad" es definida en La religión dentro de los límites de la mera razón como una "receptividad al respeto por la ley moral". Así, la disposición moral no coincide con el respeto a la ley moral pues se trata de un impulso natural previo a ese sentimiento y "facilitador" del mismo. Véase: RGV AA, VI, 028.

${ }^{15}$ Cf. Brandt (2003: 122). Sobre la distinción entre moral-jurídica (derecho) y ética, véase: Terra (2005).
} 
Debe haber en la especie humanaalguna experiencia que, como hecho (Begebenheit), indique una cualidad (Beschaffenheit) y una facultad de esta especieque sería la causa del progreso hacia lo mejor (puesto que debe ser el acto de un ser dotado de libertad),el autor del mismo; pero se puede predecir que un hecho es efecto de una causa dada cuando se producen las circunstancia que concurren a ello. SF AA, VII, 084.

En el pasaje precedente se evidencia que la prueba de la posibilidad del progreso contiene, precisamente, dos elementos centrales: lo que a través de una cierta experiencia se debe probar es 1) una cualidad o un talante (Beschaffenheit) o una facultad del género humano hacia lo mejor que actúa cuando 2) concurren las circunstancias para ello. Esto significa que el desarrollo histórico depende de una concepción de la naturaleza humana en la cual actúan oportunamente ciertos dispositivos tendientes a fin, en este caso, una facultad o talente que posteriormente, como veremos, será designada directamente como "disposición moral". Como indicamos en la Introducción y veremos más abajo, estos dos elementos: 1) ciertas "disposiciones" humanas que se desarrollan en 2) determinados contextos o circunstancias externas definen la concepción de la historia -que llamamos "inmanente"- que Kant defiende también, paradigmáticamente en Idea (y en todos sus textos previos,con excepción de Hacia la paz perpetua, tesis que aquí no podremos probar en toda su extensión). ${ }^{16}$

Kant expone en unas páginas que se volverán célebres el hecho que hará las veces de "signo histórico" buscado. Reproducimos aquí parcialmente este pasaje:

Este hecho no consiste en acciones u omisiones buenas o malas, importantes, realizadas por los hombres [...] No: nada de esto. Se trata sólo de la manera de pensar (Denkungsart) de los espectadores, que se trasluce públicamente en ese juego degrandes revoluciones [...] y que manifiesta, sin embargo, una participación (Teilnehmung) tan general y a la vez tan desinteresada por los actores de un partidorespecto de los del otro, demostrando así (a causa de launiversalidad) una carácter de la humanidad en general y, también (a causa del desinterés) un carácter moral dela misma, al menos en su disposición, que no sólo permite esperar un progreso hacia lo mejor, sino que constituye él mismo un progreso, en la medida en que la facultad para el progreso es alcanzada ahora. SF AA, VII, 085. Las itálicas son añadidos nuestros.

El signo o la experiencia que se busca no es una acción o la omisión de una acción, ni tampoco, por lo tanto, la puesta en escena de la intención de un actor o un conjunto de actores. Consecuentemente, el hecho en cuestión no es el acto revolucionario mismo. ${ }^{17}$ Kant señala

\footnotetext{
${ }^{16}$ En Hacia la pazperpetua Kant defiende un modelo exclusivamente trascendente del progreso histórico, perspectiva que está presente en todos los otros textos, aunque de manera simultánea e intercalada (de una manera que exige una clarificación que no podemos dar aquí) con una concepción "inmanente". Esto no significa que defendamos que Kant haya propuesto en 1795 una concepción "novedosa" de la historia, como decíamos, esta visión está también presente en el resto de sus textos, incluso, como vimos arriba, en El conflicto, aunque los acentos sean diferentes.

${ }^{17}$ De este modo, no consideramos acertadas las interpretaciones que sostienen que la revolución francesa es el signo histórico buscado. Esta interpretación se encuentra, sin embargo, defendida por varios intérpretes (Axinn 1971: 426; Brandt 2003: 123/6, 129; Cohen 2009: 133; Kuehn 2009: 70; Flickschuh 2009: 109). De nuestra opinión es: Beck (1791: 419) Kleingeld (1995: 77); Recki (2005: 240), Gonzalez (2014: 282), Rodriguez Aramayo (2016: 404). Nótese que Kant no se pronuncia en "Reiteración de la pregunta" a favor de la revolución, por el contrario, expresamente señala que la misma "siempre es injusta (ungerecht)". AA, VII, 088. La primera condena (moral), por parte de Kant, al acto revolucionario se encuentra en Teoría y práctica(en el pasaje: TP AA, VIII, 299-300). Con todo, a comienzos de la década de los 90's corría el rumor en Europa de que Kant defendía la revolución. E mismo se deja constatar, por ejemplo, en la carta de Biester a Kant, editor de la Berlinische Monatsschrift, a donde se publica, precisamente, Teoría y práctica, quien, luego de recibir el opúsculo, le declara a Kant que le "complació máximamente [Teoría práctica] puesto que refutaba el rumor [...] según el cual [el filósofo] se había resuelto a favor de la cada vez más repulsiva revolución francesa". Br AA, XI, 456. Los corchetes son añadidos nuestros. Ahora, la condena (moral) por parte de Kant de la revolución y su simultáneo entusiasmo por la misma -que se infiere del párrafo transcripto aquí arriba- ha conducido a algunos intérpretes a distinguir la posición pública de la verdadera posición de Kant respecto a la revolución, la última de las cuales habría sido cautelosamente ocultada por el filósofo a fin de evitar problemas con la censura. Esta interpretación es defendida paradigmáticamente en: Strauss (1988 [1952]: 33); Vaughan (1939: 83). Sin embargo, puesto que esta interpretación implica a Kant en una suerte de "mentira noble" no puede ser aceptada. Véase, por ejemplo, el artículo Anuncio de un cercano término de un tratado hacia la paz perpetua en filosofía (VNAEF AA, VIII, 411-422) en donde Kant niega enfáticamente la legitimidad de mentir en filosofía. Lewis B. Beck (1971: 411) refuta también esta lectura a través de la regla de prudencia filosófica que Kant le habría expresado a Mendelssohn en carta del 8 de abril de 1766 según la cual, aunque "habría muchas cosas que jamás tendr[á] el valor de decir, jamás diría algo en la que no cre[a]" (Br AA, X, 069. Los corchetes son añadidos nuestros) que el filósofo repite,
} 
rápidamente que dicho acontecimiento es la expresión pública de un hecho del pensamiento, eso es, dela "manera de pensar" de los espectadores de la revolución francesa. La expresión pública y generalizada a favor del bando republicano señala que los hombres tienen un interés en la defensa de constituciones populares, las cuales, además de ser aquellas en donde el pueblo es libre políticamente, son aquellas en las cuales, "por principio", se evita la guerra agresiva, se trata de aquella constitución que "es justa y moralmente buena en sí misma" (SF AA, VII, 085). Los espectadores externos de la revolución no son imparciales en la contemplación del acontecimiento, por el contrario, toman parte, aunque desinteresadamente, por el lado que alberga la causa moral, es decir, por el bando republicano. Esto significa que ellos expresan una disposición moral de todo el género humano. En los términos de Kant:

La revolución de un pueblo lleno de espíritu, que hemos visto realizarse en nuestros días [...] encuentra en los espíritus de todos los espectadores (que no están comprometidos ellos mismos en este juego) una participación según el deseo (Wunsche), rayana al entusiasmo (Enthusiasm), y cuya manifestación, que lleva aparejado un riesgo, no podía obedecer a otra causa que una disposición moral del género humano. SF AA, VII, 085.

De este modo, se prueba a través de un indicio, esto es, la manifestación de un afecto cercano a una pasión, el entusiasmo, ${ }^{18}$ que el género humano tiene una disposición moral. El verdadero entusiasmo es "una participación con afecto en el bien" y "se refiere siempre a lo ideal y lo puramente moral, esto es, al concepto de derecho" (SF AA, VII, 086). Puesto que es un afecto que nace de la idea pura de derecho parece ser un candidato adecuado para expresar que el género humano tiene una tendencia a desarrollar en la historia aquello que es deber moral para la especie. Esto significa que el progreso histórico se comprende en El Conflicto a partir de un dispositivo inmanente de la naturaleza humana, orientado a fin -la disposición moral- que opera, como veremos inmediatamente, según la ocasión.

Pues bien, si hay una disposición moral en el género humano, ¿por qué es, entonces, la historia el escenario de la opresión interna y la guerra externa? Si bien es cierto que Kant afirma que puede haber progreso en la medida en que es "una circunstancia que él [el hombre] mismo -mediante su buena voluntad- puede realizar" (SF AA, VII, 084) eso no significa que aquella "disposición moral" pueda realizarse en cualquier momento o circunstancia. Las circunstancias del progreso dependen de una disposición moral... pero la actualización de dicha disposición depende, a la vez, de que ocurran ciertas circunstancias. ¿Cuáles son esas circunstancias? En la sección quinta Kant señalaque las mismas "tienen que ocurrir" alguna vez. Como en el "cálculo de probabilidades en el juego de azar" (SF AA, VII, 084) se puede afirmar que dichas circunstancias ocurrirán probablemente alguna vez, aunque no se pueda determinar con precisión cuándo esto vaya a suceder. En la sección séptima Kant nos asegura que por los signos de la época la especie humana logrará algún día el fin esperado y que "desde ese momento su progreso hacia lo mejor nunca reconocerá una regresión total" (SF AA, VII, 088). Nuevamente aquí indica que "en lo que se refiere al momento, (podemos predecir dicho acontecimiento) solo de una manera indeterminada" (SF AA, VII, 088). Sin embargo,aunque asegura una y otra vez el requerimiento de que ciertas circunstancias co-actúen junto a la disposición moral en el desarrollo histórico, nunca define ni deja en claro de cuáles se trata. Lo importante para nosotros es notar que este factor apunta a mostrar que la disposición implicada no se desarrolla por sí misma y en aislamiento, sino que depende siempre de un contexto propicio para su desenvolvimiento.

nuevamente, en 1798 (en El conflicto, véase: SF AA, VII, 032). Beck (1971: 413) resuelve la aparente paradoja de la posición de Kant respecto a la revolución mediante la distinción entre un rechazo "jurisprudencial" al derecho a la revuelta (o revolución) y una simpatía "no jurisprudencial" hacia la misma. Puesto que la posición negativa se localiza en un nivel lógico diferente a la posición positiva, las mismas no se contradicen.

${ }^{18}$ Para el concepto de "entusiasmo" (Enthusiasm, Enthusiasmus o Begeisterung), véase, fundamentalmente: KU AA, V, 271/2, además: NTH AA, I, 329; GSE AA, II, 221, 251, 253, 267; KU AA, V, 157, 272, 274, 275, 363; RGV AA, VI, 050, 195; MS TL AA, VI, 409; SF AA, VII, 085, 086; Anth AA, VII, 202, 254, 259, 269, 314; TP AA, VIII, 287. Se ha ocupado especialmente de la cuestión del "entusiasmo revolucionario" en tanto forma de lo sublime en Kant Jean-François Lyotard (1986). 


\section{Sobre la novedad de la concepción kantiana de historia en 1798}

Según veremos en el presente apartado, antes de 1798, paradigmáticamente en Idea, Kant defiendeuna concepción inmanente del desarrollo histórico. Esto es, una visión que da cuenta del progresoa partir de la idea de que ciertas "disposiciones" internas de la especie humana se despliegan segúnciertas "circunstancias externas". Así se debe admitir una continuidad en el marco teórico general con el que Kant concibe el desarrollo histórico en los años 1784 y 1798, inclusive.Ahora, esto no significa que la concepción kantiana de la historia no sufra cambios significativos. De hecho, como analizaremos abajo, en Idea, el peso del argumento del progreso está en mostrar que las disposiciones no morales del hombre dirigen a la especie hacia un ámbito moral y no, como en $E l$ conflicto, en probar, directamente,la existencia de una disposición moral.Según Idea se inscriben en la naturaleza humana disposiciones no morales de las que surgen pasiones cuya tensión produce mecanismos de transformación cultural que, a la larga, conducen a toda la especie humana, incluso sin quererlo,a la institución de un orden legal interior y exterior, perfectamente justo, en donde se puede desarrollar la disposición moral de los hombres. Así, el desarrollo de la disposición moral depende en el texto de 1784, como mostraremos a continuación,del desarrollo de dispositivos no morales.

De esta manera, en Idea Kant inaugura la problemática de la historia universal mediante los conceptos de "disposiciones originales" o "disposiciones naturales", que son centrales entre las tesis primera y cuarta. ${ }^{19}$ En la primer tesis el filósofo afirma que todas las disposiciones de un ser orgánico han de desarrollarse en algún momento de manera completa y conveniente. ${ }^{20}$ En la segunda tesis añade, sin embargo, que puesto que las disposiciones destinadas al uso de la razón en el hombre se desarrollan de forma ilimitada y que los seres humanos son mortales, las primeras no se desarrollan de forma completa en cada individuo, sino sólo en la especie. Estas dos tesis son las premisas de la afirmación hacia el final de la tesis tercera cuando asegura que es necesaria la idea de que las generaciones pasadas de la humanidad deban laborar para la fortuna de las generaciones futuras.

Pues bien, en la tesis cuarta de Idea Kant se ocupa de comprender el tránsito desde el estado de barbarie de la especie hacia la cultura ${ }^{21}$ a partir del concepto de "disposición" inscripta en la "naturaleza humana". Las disposiciones juegan aquí un doble papel pues no sólo son aquello que se debe desarrollar en el tiempo, sino también el medio o la circunstancia mediante el cual las mismas se desarrollan. Así, el paso de la animalidad hacia la humanidad del hombre es comprendido mediante el "antagonismo de las [disposiciones] dentro dela sociedad" del que finalmente resulta un ordenamiento legal que es condición, a la vez, para el desarrollo de "todas las disposiciones" de la especie. ${ }^{22}$ Veamos:

El medio del que se sirve la Naturaleza para llevar a cabo el desarrollo de todas sus disposiciones es el antagonismo de las mismas dentro de la sociedad, en la medida que ese antagonismo acaba por convertirse en la causa de un orden legal de la misma. IaG AA, VIII, 020.

Kant señala inmediatamente a continuación que el "antagonismo de las disposiciones dentro de la sociedad" es equivalente al concepto de "insociable sociabilidad" (ungesellige Geselligkeit) que, según defiende aquí Kant, es un conjunto de disposiciones que se reducen a la "propensióna vivir en sociedad, vinculada con una resistencia continua" ${ }^{23}$ a entrar en la misma. ${ }^{24} \mathrm{La}$ "insociable sociabilidad" está compuesta, así, por un lado, por una disposición social del hombre a reunirse en sociedad, esto es, una inclinación a estar en compañía de los otros y, por otro lado, por la disposición

${ }^{19}$ Reponemos aquí parte de lo que hemos desarrollado en nuestro artículo: Lerussi, 2015

${ }^{20} \mathrm{IaG}$ AA, VIII, 018

${ }^{21}$ Una definición amplia de "cultura" se encuentran en: KU AA, V, 430, 431

${ }^{22}$ Véase: IaG AA, VIII, 022.

${ }^{23}$ Véase: IaG AA, VIII, 020

${ }^{24}$ Hemos desarrollado nuestra interpretación del concepto de "insociable sociabilidad" en: Lerussi (inédito). 
insociable que consiste en una inclinación a aislarse y a vivir según el propio sentido. ${ }^{25}$ Así, ni a la disposición sociable ni a la insociable se le adscribe una connotación moral. Por el contrario, refieren, respectivamente, a una inclinación o a un rechazo hacia la compañía de los otros y no a un deseo de hacerles el bien o hacerles daño, de competir o cooperar con ellos. Ahora bien, la disposición a aislarse degenera tan pronto se pone en tensión con la disposición sociable, pues seres que tienen la inclinación a vivir según el propio modo de pensar querrán continuar haciéndolo cuando se encuentren, por el principio de la sociabilidad, en comunidad con los otros. Por eso, se dice en Idea que el hombre en sociedad se encuentra en un permanente estado de alerta, ya que "como se sabe inclinado a oponerse a los demás, espera hallar esa misma resistencia por doquier". ${ }^{26} \mathrm{El}$ hombre en sociedad se resiste frente a los demás porque sabe que los demás querrán, como él, vivir según su propio criterio en sociedad. Más aún, es la presunción de que los otros pretendan imponerle su propio sentido o modo de pensar lo que inclina al hombre a entrar, en una carrera por el rango entre sus congéneres, a un estado de competencia por obtener los medios que otorgan superioridad al hombre y que facilitan la imposición del propio sentido. Es a partir de esta competencia por el rango de donde surge una tríada de pasiones que implican las tres formas centrales de competencia entre los hombres, esto es, la competencia por la riqueza, por el dominio y por el honor. Así, mediante la "ambición, el afán de dominio o la codicia" se produce, finalmente, "los primeros pasos desde la barbarie hacia la cultura". ${ }^{27 "}$ Aunque por el principio a la sociabilidad, el hombre quiere vivir "cómoda y placenteramente", en "laxitud y ocioso conformismo", por su disposición a la insociabilidad en el contexto de la sociedad debe salir de la indolencia y del inactivo contento, "entregándose al trabajo y padeciendo las fatigas que sean precisas para encontrar prudentemente los medios de sustraerse de tales penalidades." ${ }^{28}$ De este modo, mediante los frutos del trabajo fatigoso, riquezas, dominio y honores, el hombre puede encontrar una ventaja en relación a sus congéneres y, por consiguiente, facilitar la imposición del propio sentido o modo de pensar.

Pues bien, en este esquema de desarrollo de la cultura a través del antagonismo de las disposiciones no morales las circunstancias externas también juegan un papel. Como señala el filósofo,el desarrollo de ciertas disposiciones produce un estado de cosas (A) -que Kant denomina "tensión de fuerzas" (Anspannung der Kräfte) ${ }^{29}$ que despierta disposiciones aún dormidas cuyo desenvolvimiento dará en un nuevo estado de cosas (B) - "nueva tensión de fuerza"- que será punto de partida para el desarrollo de nuevas tendencias.Entre la tesis cuarta y octava de Idea se destacan una serie de factores externos, "relaciones de fuerza" o circunstancias culturales mediante las cuales son promovidas diferencialmente las disposiciones naturales de los hombres. Significativamente, en la sección cuarta, quinta y octava de Idea, Kant presenta una concepción del progreso histórico según la cual el paso de cada circunstancia cultural a la siguiente, esto es, de la barbarie a un orden legal, de éste, a una sociedad civil plenamente justa y, finalmente, de ésta, a una sociedad moral en donde pueden desarrollarse todas las disposiciones humanas, se produce a partir de las condiciones generadas por las circunstancias culturales previas.

En la tesis cuarta de Idea, Kant indica abruptamente que el efecto que tendrían ciertos productos de la cultura, es decir, del arte y la ciencia, es producir un cierto sentimiento (la formación de gusto) y un nuevo modo de pensar, en general. ${ }^{30}$ Una sociedad patológicamente formada en la que los hombres compiten por producir cultura, a la larga, a través de la apreciación y discusión pública de sus productos, hace nacer una nueva afectividad y modo de pensar que "andando el tiempo,puede transformar las toscas disposiciones naturales hacia la disquisición moral en principios prácticos

${ }^{25}$ Véase: IaG AA, VIII, 020/1.

${ }^{26}$ Véase: IaG AA, VIII, 021.

${ }^{27}$ Véase: IaG AA, VIII, 021

${ }^{28}$ Véase: IaG AA, VIII, 021.

${ }^{29}$ Véase: IaG AA, VIII, 019

${ }^{30}$ Véase: IaG AA, VIII, 021. 
determinados", que es base de una "sociedad moral". ${ }^{31}$ De este modo, una sociedad del gusto y de la ilustración, resultante del estado de competencia, tendría por consecuencia, la moralidad y el consecuente desarrollo de todas las disposiciones humanas, esto es, incluso la disposición moral. ${ }^{32}$

Así, en 1784 Kant defiende -como en El conflicto- una concepción inmanente de la historia, aunque aquíla función explicativa del desarrollo histórico está del lado de las disposiciones no morales (disposiciones sociable e insociable); el desarrollo de la disposición moral es, por el contrario, aquello que tiene que ser explicado y es resultado del progreso histórico. Hacemos notar brevemente que el devenir histórico se entiende, así, en este contexto, como un procesosucesivo y continuo de formas culturales o civilizatorias (sociales, político-jurídicas y afectivas) cuyas tensiones internas producen nuevas formas culturales que, a la larga y por acumulación, produciría un estado en donde los hombres pueden desarrollarse moralmente. El esquema de progreso histórico implicado en El conflicto parece ser muy diferente.

Según vimos, en 1798 Kant propone una línea argumental en la que las disposiciones no morales no tienen ninguna función explicativa en la comprensión del progreso humano. Por el contrario, el progreso se comprende a partir de la puesta al descubierto de que hay en el género humano una disposición moral que se despliega cuando concurren las circunstancias para ello. ${ }^{33}$ Este giro en la argumentación tiene, a la vez, consecuencias en la concepción de la estructura de la historia pues Kant parece abandonaren 1798 la concepción del progreso como proceso acumulativo de formas culturales o civilizatorias. Esta vez, "progreso" significa la institución de una constitución en la cual el pueblo se da su propia ley y evita la guerra (agresiva) contra otros Estados. Kant señala en $E l$ conflicto que la prueba del progreso del género humano hacia lo mejor no sólo está orientada al futuro, sino que también "podría ser extendida a la historia de los tiempos pasados", esto es, "que siempre ha habido progreso" (SF AA, VII, 084), sin embargo, esto no implica que él entienda también aquí por "progreso" un continuum lineal e incesante del mejoramiento moral de la especie humana desde los primeros tiempos hasta el presente. "Siempre ha habido progreso" significa, por el contrario, que en todos los tiempos ha sido posible para los pueblos, dadas las circunstancias, republicanizarse. El "progreso" no implica como antes la acumulación de experiencias pasadas, sino la irrupción impredecible de la causa moral en la historia.

\section{Bibliografía}

Primaria

KANT, Immanuel: Kantsgesammelte Schriften, Berlin, KöniglichPreussischen Akademie der Wissenschaften, 1900ss.

Ediciones en español

KANT, Immanuel: "Idea para una historia universal en clave cosmopolita", ¿Qué es la Ilustración?, traducción de R. Rodriguez Aramayo y Concha Roldán Panadero, Madrid, Alianza, 2011.

\footnotetext{
${ }^{31}$ Véase: IaG AA, VIII, 021.

${ }^{32}$ Esta tesis se repite en: KU AA, V, 433ss.

${ }^{33} \mathrm{La}$ circunstancia de que el rol del explanans del progreso histórico recaiga en El conflicto sobre la disposición moral y no, como antes, en las disposiciones no morales permite quizás iluminar, al menos en parte, por qué -como han señalado tanto Brandt (2003) como Perez $(2006,2014)$ - Kant hace (mayor) uso de la figura de la "intención de la naturaleza" o "providencia" en los textos que van desde 1784 (Idea) a 1795 (Hacia la paz perpetua). Esto es así, sugerimos, porque las disposiciones no morales son características de la naturaleza humana que actúan tengan o no los hombres conciencia de ellas, el agente es aquí la naturaleza (humana) "no-consciente". En 1798, en cambio, puesto que la agencia histórica corre del lado de la disposición moral, el acento es puesto en los efectos conscientes de la naturaleza humana, esto es, en la acción colectiva y organizada de los hombres.
} 
[Hemos introducido variaciones en esta traducción cuando lo hemos considerado pertinente].

KANT, Immanuel: "Teoría y práctica: En torno al tópico: esto vale para la teoría, pero no sirve para la práctica", traducción de Roberto Rodríguez Aramayo y Francisco Pérez Lopez, Madrid, Alianza, 2011.

KANT, Immanuel: La religión dentro de los límites de la mera razón, traducción de Felipe Martínez Marzoa, Madrid, Alianza, 1969.

KANT, Immanuel: El Conflicto de las facultades, traducción de Elsa Tabering, Bs. As., Losada, 1963. [Hemos introducido variaciones en esta traducción cuando lo hemos considerado pertinente.]

Literatura crítica

ARENDT, Hannah:Lectures on Kant's Political Philosophy, edited and with an interpretative Essay by Ronald Beiner, Chicago, The University of Chicago Press, 1982. Los textos citados provienen de la traducción de Carmen Corral: Lecciones sobre la filosofía política de Kant, Madrid, Paidos, 2003.

AXINN, Sidney: "Authority, and the French Revolution”, Journal of the History of Ideas Vol. 32 Nro. 3 (1971) 423-432.

BECK, Lewis. W.: "Kant and the Right of Revolution”, Journal of the History of Ideas Vol. 32 Nro. 3 (1971) 411-422.

BRANDT, Reinhard: Universität zwischen Selbst- und Fremdbestimmung. Kants >Streit der Fakultäten<, Berlin, Akademie Verlag, 2003.

COHEN, Alix: Kant and the Human Sciences. Biology, Anthropology and History, Hampshire, Macmillan, 2009.

FLIKSCHUH, Katrin: "Duty, nature, right: Kant's response to Mendelssohn in Theory and Practice III", Journal of Moral Philosophy, 4 (2), 2007, pp. 223-241.

FLIKSCHUH, Katrin: "Hope or prudence? Practical faith in Kant's political thinking", en Stolzenberg, Jurgen y Rush, Fred (eds.), Faith and Reason. International yearbook of German idealism 7, De Gruyter, Berlin/New York, 2009.

FOUCAULT, Michel, Qu'est-ce que les lumières?, Paris, Breal, 2004 [1983]. Se usa la traducción al español de Silvio Mattoni: “¿Qué es la ilustración?”, Córdoba, Alción, 1996.

GERHARDT, Volker: "Kulturelle Evolution. Philosophische Anmerkungen zu einem nicht erst seit Darwin aktuellen Programm", en GERHARDT, Volkery NIDA-RÜMELIN, Julian (ed.): Evolution in Natur und Kultur, Berlin, De Gruyter, 2010.

GERHARDT, Volker: Immanuel Kant. Vernunft und Leben, Stuttgart, Reclam, 2002.

GONZALEZ, Ana Marta, "Kant on History”, Estudos Kantianos, V. 2, N. 2, p. Jul./Dez. 2014, pp. 265-290.

KLEINGELD, Pauline: Fortschritt und Vernunft: Zur Geschichtsphilosophie Kants, Würzburg, Königshausen \& Neumann, 1995.

KUEHN, Manfred:"Reason as a species characteristic", en RORTY, Amélie Oksenberg y SCHMIDT, James (eds.), Kant's Idea for a Universal History with a Cosmopolitan Aim, Cambridge, Cambridge University Press, 2009, 68-93.

LEBRUN, Gérard: Kant et la fin de la métaphysique. Essai sur la Critique de la faculté de juger, Paris, Armand Colin, 1970.

LEBRUN, Gérard: “Une teléologie pour l'histoire? La première proposition de l'Idée d'une histoire universelle", en CLAVIER, Paul y WOLFF, Francis (eds.), Kant sans kantisme, Paris, Fayard, 2009. 
LERUSSI, Natalia: "Acerca de una consideración naturalizada de la filosofía de la historia de Immanuel Kant. Epigénesis e historia universal”, Cadernos de Filosofia Alemã, Vol. 20, Nro. 1, 2015, pp. 93-105.

LERUSSI, Natalia: "Unsocial Sociability, Human Nature and Evil", presentado en el marco del VI KANT MULTILATERAL COLLOQUIUM: Kant on Violence, Revolution, and Progress: Historical, Political, and Metaphysical Themes, organizado por la Hofstra University, New York, 7-9 de agosto de 2016, inédito.

LYOTARD, Jean-François: $L^{\prime}$ enthousiasme, Paris, Galilée, 1986. Hay edición en español: El entusiasmo. Crítica kantiana de la historia, Barcelona, Gedisa, 1994.

PEREZ, Daniel Omar: “Os significados da história em Kant”Philosophica 28 (2006) 67-107.

PEREZ, Daniel Omar: "Discussão. História e teleologia na filosofia kantiana. Resposta às críticas de Ricardo Terra contra a «Escola semántica de Campinas»”, Studia kantiana 16 (2006) 144159.

RECKI, Birgit: "Fortschritte als Postulat und die Lehre von Geschichtszeichnen", en GERHARDT, Volker (ed.): Kant im Streit der Fakultäten, Berlin/New York, De Gruyter, 2005.

RODRIGUEZ ARAMAYO, Roberto, "Right as a Sign of a Philosophical Chiliasm: Freedom and its Evolution in Kant's Opuscules", en VV.AA., Critical Paths outside the Critiques. Kant's Shorter Writings, Cambridge Scholar Publishing, New Castle, 2016.

STRAUSS, Leo: Persecution and the Art of Writing, Chicago/London, The University of Chicago Press, 1988, [1952]. Hay edición en español: La persecución y el arte de escribir, Madrid, Amorrortu, 2009.

TERRA, Ricardo: “A distinção entredireito e ética na filosofía kantiana”, en PEREZ, Daniel Omar (ed.), Kant no Brasil, San Pablo, Editora Escuta, 2005.

TERRA, Ricardo: "História e Direito em 1784. Comentários sobre a interpretação da «Escola Semântica de Campinas»", Studia kantiana 12 (2002) 175-194.

VAUGHAN, C. E.: Studies in the History of Political Philosophy before and after Rousseau, Manchester, A.G. Little, 1939.

WOOD, Allen: "Unsociable Sociability: The Anthropological Basis of Kantian Ethics", Philosophical Topics 19:1 (1999) 325-351.

WOOD, Allen: "Kant's Fourth Proposition: the unsociable sociability of human nature", en RORTY, Amélie Oksenberg y SCHMIDT, James (ed.), Kant's Idea for the Universal History with a Cosmopolitan Aim. A Critical Guide, New York, Cambridge University Press, New York, 2009. 